3 Owen D, Davidson J. Hubris syndrome: an acquired personality disorder? A study of US Presidents and UK Prime Ministers over the last 100 years. Brain 2009; 132: 1396-406.

4 Gureje O. Nosological changes in psychiatry: hubris and humility. World Psychiatry 2012; 11: 28-9.
5 Vogelstein E. Professional hubris and its consequences: why organizations of health-care professions should not adopt ethically controversial positions. Bioethics 2016; 30: 234-43.

\section{psychiatry in literature}

\section{Reflection on: Madness and Modernism: insanity in the light of modern art, literature, and thought (revised edition)}

\section{Giovanni Stanghellini}

We need a psyche for psychiatry. Madness and Modernism is an exemplary essay in this sense. Louis Sass's book (first published in 1992, now in a revised edition from Oxford University Press) is a work of comparative phenomenology: it gives attention to two domains: one is madness, or more exactly schizophrenic subjectivity; the other is the modernist or avant-gardist orientation in culture and the arts that came to prominence around 100 years ago. The main purpose of matching these two domains is to use modernism as a way of illuminating madness. Through the analyses of modernist (and so-called postmodernist) style and sensibility, and its cultural and aesthetic characteristics, one distinctive property is brought to the fore: hyperreflexivity, the exaggerated tendency to direct focal and explicit attention to the otherwise implicit background of experience. Hyperreflexivity, the rendering-explicit of the implicit conditions of possibility of experience, has a revelatory power in the arts (for instance, in Magritte's surrealist paintings) and in culture (for instance, in phenomenological philosophy), as it uncovers aspects of human experience that would otherwise go unnoticed.

Yet it also has an alienating power, since it involves detachment from common-sense experience and disengagement from everyday pragmatic concerns, and even from one's own bodily reality - often inspiring an unusual interest for theoretical or metaphysical questions about the 'reality of reality' or the 'secret workings of the psyche'- or what one of my intellectually oriented patients termed 'what remains hidden to the majority beyond Maja's veil'.

Having identified hyperreflexive awareness as a key feature of modernism (one that offers a unifying vision of otherwise disparate cultural and artistic trends), this construct can be used to shed light on the experiential dimension of certain forms of alienated consciousness in mental disorder - where the reflexivity in question may often have a more automatic, afflictionlike quality. In this way, it offers rare insight into both altered modes of experience (for example of space, time and language) and the personal meaning that patients often attach to these experiences. This is obviously a great achievement for all those who believe that psychiatry is first and foremost, although not exclusively, about understanding 'what it is like' to suffer from abnormal mental conditions.

Developments in psychiatric research have certainly extended our knowledge about the causes of mental disorders, but mental disorders have meanings in addition to causes; they involve alterations of experience as well as of the brain and nervous system. Of course, this project - which is that of phenomenological psychopathology - is a very different research programme for psychiatry than that of neuroscientific research. The two programmes are not incompatible, however, since in order to correlate any given abnormal experience to brain functions, one must first be able to define such experiences with sufficient precision. We should remember that we as psychiatrists do not sit in front of a broken brain - we confront a suffering person. Indeed, mental disorders just are primarily disorders of the human psyche. If a crucial task of psychiatry is to understand abnormal human existence, then psychiatry needs to consider psychopathological conditions in terms of basic anthropological categories that capture the a priori norm or framework within which a given phenomenon of human existence occurs. Some philosophers and psychopathologists have already taken this direction. One of the first was Hegel, who sensed that 'something akin to philosophy' was often struggling within madness -indeed, Hegel defined psychosis as the unhealthy twin of authentic idealism. 'Double bookkeeping' (Bleuler), the understanding of schizophrenic autism as loss of common sense (Blankenburg) and 'epistemological delusions' (Sass) demonstrate philosophy's ability to help us re-think certain psychopathological conditions, and to alter some of our most taken-for-granted assumptions (such as the notion that schizophrenic delusion is necessarily a matter of 'poor reality-testing').

This is the contribution that human sciences can bring into the field of psychiatry. Yet the rapport between psychiatry and the human sciences can be reciprocal. Philosophy and the humanities can contribute to the understanding of mental disorders, but abnormal mental conditions can contribute to the understanding of human existence - and this by revealing, in starkest terms, some key potentialities and paradoxes of the human mind. In this vein, philosopher Paul Ricoeur has developed the concept of 'importance' - which refers to the way a phenomenon's meaning may exceed or transcend its initial context. An important phenomenon reveals meanings that can be actualised in situations beyond the one in which it occurred. We as psychiatrists are locally responsible for caring for the individual person who seeks help. We may, however, also have a certain more global responsibility for considering how a patient's condition can shed light on human existence as such. The unavoidability of guilt, inescapability of freedom or loneliness of one's existence; the fragility of one's body or sense of reality: these are but a few examples of fundamental human issues that abnormal mental conditions both exemplify and reveal. Psychiatrists, along with knowledge about the brain, should also be ready to explore such issues to illuminate the feelings and meanings of their patients. Madness and Modernism, certainly one of the outstanding books illuminating madness written in the last century, is an exceptionally rich source of ideas and materials for such a project.

Copyright (C) The Royal College of Psychiatrists 2018 